Revista

Ibero-Americana

de Estratégıa

\title{
ESTRATÉGIA COOPERATIVA EM REDES ORGANIZACIONAIS: UM ESTUDO EM POLO TURÍSTICO DE MINAS GERAIS
}

COOPERATIVE ORGANIZATIONAL NETWORKS STRATEGY:

A STUDY IN THE TOURISM CENTER, MINAS GERAIS, BRAZIL

ESTRATEGIA COOPERATIVA EN REDES ORGANIZACIONALES:

UN ESTUDIO EN EL POLO TURÍSTICO DE MINAS GERAIS

\section{Maximiliano Francisco Oliveira}

Mestre em Administração pela Universidade FUMEC (Fundação Mineira de Educação e Cultura) Especialista em Gestão Estratégica pelo Centro de Pós-Gradução em Administração da Universidade Federal de Minas Gerais - CEPEAD/UFMG

E-mail: max.bh@terra.com.br (Brasil)

\section{Carlos Alberto Gonçalves}

Doutor em Administração pela Universidade de São Paulo - USP

Professor do Programa de Mestrado e Doutorado da Universidade FUMEC (Fundação Mineira de Educação e Cultura)

E-mail: carlos@ face.ufmg.br (Brasil) 
Estratégia Cooperativa em Redes Organizacionais: Um Estudo em Polo Turístico de Minas Gerais

\title{
ESTRATÉGIA COOPERATIVA EM REDES ORGANIZACIONAIS: UM ESTUDO EM POLO TURÍSTICO DE MINAS GERAIS
}

\section{RESUMO}

Esse trabalho objetivou descrever a estrutura de relacionamento em redes entre as pousadas localizadas na Serra do Cipó - MG. Para o desenvolvimento da análise, procurou-se verificar a existência, a intensidade, a potencialidade, bem como a relação dos seguintes construtos: atuação em redes, cooperação, aprendizagem relacional e fragmentação da rede. Os dados foram coletados por meio da aplicação de um questionário e tratados com o software Ucinet 6.0, apoiado na teoria de análise de redes sociais. Foram utilizadas também técnicas de análise de regressão estatística e da estatística descritiva. Os resultados iniciais apontaram a presença de todos os construtos analisados, com destaque para a atuação em redes que atingiu a maior intensidade, seguida, sucessivamente, pelo aprendizado relacional e pela fragmentação da rede. A cooperação foi o quesito com a menor intensidade, porém, com a maior coesão. Com base nos testes do modelo hipotético proposto, podese verificar a significância da relação positiva entre os construtos: atuação em redes e cooperação, cooperação e aprendizagem relacional. A relação entre aprendizagem relacional e fragmentação da rede também foi significativa, porém, de forma negativa e com baixo coeficiente de determinação. A partir das conclusões, foi possível confirmar a premissa inicial da atuação em redes entre as empresas estudadas; verificar a presença da cooperação na estrutura de relacionamento, apesar do subaproveitamento do seu potencial; e constatar a busca de aprendizado relacional na cadeia cooperativa.

Palavras-chave: Aprendizado Relacional; Estratégia Cooperativa; Redes Organizacionais.

\section{COOPERATIVE ORGANIZATIONAL NETWORKS STRATEGY:}

\section{A STUDY IN THE TOURISM CENTER, MINAS GERAIS, BRAZIL}

\begin{abstract}
The work at hand consists in an analysis of the relationship structure amongst the inns located in "Serra do Cipó", Minas Gerais, Brazil. It aims to describe how this structure determines cooperation and relational learning. In order to develop the analysis, researchers verified the existence, the intensity, the potentiality and the relation of the following constructs: actuation networks, cooperation, relational learning and network fragmentation. The data were collected through the application of a questionnaire and were tested through the software Unicet 6.0 that analyses social networks. Techniques of analysis of statistical regression and techniques of descriptive statistics were also used. The earlier results showed the presence of all the analyzed constructs, with an emphasis on actuation networks that reached the highest intensity, followed successively by relational learning and network fragmentation. Cooperation was the construct that presented the lowest intensity, however, with highest cohesion. Based on tests of the proposed hypothetical model, the significance of positive relation amongst these constructs can be verified: actuation networks and cooperation, cooperation and relational learning. The relation between relational learning and network fragmentation was also significant but with a negative relation. From the findings, it was possible to confirm the initial premise of the action networks between the companies studied, to verify the presence of cooperation in the structure of the relationship, despite the underutilization of its potential, and to see the search for relational learning in the cooperative chain.
\end{abstract}

Keywords: Cooperative Strategy; Organizational Networks; Relational Learning.

Revista Ibero-Americana de Estratégia - RIAE, São Paulo, v. 10, n. 2, p. 34-57, mai./ago. 2011. 
Maximiliano Francisco Oliveira \& Carlos Alberto Gonçalves

\section{ESTRATEGIA COOPERATIVA EN REDES ORGANIZACIONALES: UN ESTUDIO EN EL POLO TURÍSTICO DE MINAS GERAIS}

\section{RESUMEN}

Este trabajo tuvo como objetivo describir la estructura de la relación en redes entre los alojamientos localizados en la Serra do Cipó - MG. Para el desarrollo del análisis, se procuró verificar la existencia, la intensidad, la potencialidad, así como la relación de los siguientes constructos: la actuación en redes, la cooperación, el aprendizaje relacional y la fragmentación de la red. Los datos fueron recolectados a través de la aplicación de una encuesta y tratados con el software Ucinet 6.0, apoyado en la teoría de análisis de redes sociales. También se usaron técnicas de análisis de regresión estadística y de la estadística descriptiva. Los resultados iniciales indicaron la presencia de todos los constructos analizados, con destaque para la actuación en redes que alcanzó la mayor intensidad, seguida, consecutivamente, por el aprendizaje relacional y por la fragmentación de la red. La cooperación fue el requisito con menor intensidad, sin embargo, con la mayor cohesión. Con base en las pruebas del modelo hipotético propuesto, se puede verificar el significado de la relación positiva entre los constructos: actuación en redes y cooperación, cooperación y aprendizaje relacional. La relación entre el aprendizaje relacional y la fragmentación de la red también fue significativa, sin embargo, de una manera negativa y con bajo coeficiente de determinación. A partir de las conclusiones, fue posible confirmar la premisa inicial de la actuación en redes entre las empresas estudiadas; verificar la presencia de la cooperación en la estructura de la relación, a pesar del sub-aprovechamiento de su potencial; y constatar la búsqueda del aprendizaje relacional en la cadena cooperativa.

Palabras-clave: Aprendizaje Relacional; Estrategia Cooperativa; Redes Organizacionales. 
Estratégia Cooperativa em Redes Organizacionais: Um Estudo em Polo Turístico de Minas Gerais

\section{INTRODUÇÃO}

A globalização e os acontecimentos de natureza política, econômica, social, cultural e tecnológica alteraram o ambiente dos negócios com capilaridade e, principalmente, com grande velocidade (Gonçalves, Gonçalves Filho e Reis Neto, 2006; Peci, 1999). A competição, que antes era local e limitada, passou a se originar de qualquer parte do mundo, intensificando a luta pela sobrevivência e dificultando a realização do desempenho superior da firma. A busca pela vantagem competitiva tornou-se mais árdua e mais complexa, exigindo, por parte das empresas, níveis de serviço superiores a preços cada vez mais reduzidos. Nesse cenário, a rivalidade se prolifera, dando origem à hiperconcorrência (Hitt, Ireland e Hoskisson, 2008).

Se, para as empresas globais, os desafios de prosperar são grandes, para as micro e pequenas empresas (MPEs) são ainda maiores. Com recursos limitados e, na maioria das vezes, com pouca experiência e conhecimento, esse tipo de empresa é fortemente atingida pela hiperconcorrência. As estatísticas comprovam tal vulnerabilidade ao constatarem que 36\% das micro e pequenas empresas não sobrevivem aos quatro primeiros anos de existência (Sebrae, 2009). Além disso, levando-se em consideração que elas representam $98 \%$ do total dos negócios formais existentes no país e $94 \%$ das ocupações (Sebrae, 2009), torna-se imperativa a busca por soluções alternativas para amenizar essa situação.

Como resposta a esse desafio, surgem novas estruturas organizacionais, que, segundo Amato Neto (2000), estão ligadas à constituição de novas formas de relacionamento entre empresas, particularmente aquelas envolvendo pequenas e médias organizações. Para Peci (1999), no atual ambiente competitivo, as empresas devem se concentrar nas suas competências básicas e estabelecer parcerias com outras organizações, por meio da formação de redes organizacionais. Castells (1999) concorda com a importância da estrutura em rede e afirma que as redes são e serão os componentes fundamentais das organizações e que tal estrutura será a forma predominante de concorrência na nova economia global. $\mathrm{O}$ autor destaca ainda que as organizações tradicionais, ao operarem de forma individualizada, enfrentarão dificuldades quanto aos novos desafios.

Pelo caráter multidisciplinar dos assuntos relacionados às redes organizacionais (Matheus e Silva, 2006), são vários os conceitos e terminologias encontrados na literatura, tais como clusters (Porter, 1998), aglomerados competitivos, consórcios, sistemas produtivos locais, polos (Casarotto Filho e Pires, 2001), aglomerados territoriais, distritos industriais, cadeias produtivas (Lastres e Cassiolato, 2005) e redes interorganizacionais (Lazzarini, 2008). Mesmo com essa diversidade, grande parte dos estudos disponíveis remete a ideias similares, em que a cooperação entre os participantes da rede é o foco central. Para esse trabalho, tendo em vista o objetivo de descrever a

Revista Ibero-Americana de Estratégia - RIAE, São Paulo, v. 10, n. 2, p. 34-57, mai./ago. 2011. 
estrutura de relacionamento em rede das pousadas localizadas na Serra do Cipó, o termo aglomerado territorial será utilizado na argumentação pelo fato de sua principal característica considerar diversas empresas, do mesmo setor ou de setores afins, em uma mesma localidade geográfica (Lastres e Cassiolato, 2005; Petrocchi, 2002).

Os benefícios que um aglomerado territorial pode propiciar são vários, como, por exemplo, o acesso facilitado à matéria-prima e à mão de obra especializada (Lastres e Cassiolato, 2005); à economias de escala; ao desenvolvimento de barreiras aos novos entrantes (Ferreira Junior e Teixeira, 2007); à redução dos custos de transação; à redução de custos em pesquisas; à promoção do desenvolvimento local (Andrighi e Hoffmamm, 2008), entre outros. Entre os diversos benefícios que podem ser identificados, os que sobressaem na literatura sobre as vantagens da proximidade geográfica referem-se à disseminação da informação, à transferência do conhecimento e ao aprendizado organizacional (Balestrin e Fayard, 2003; Hoffmann, Molina-Morales e MartinezFernandez, 2004). Contudo, para que tais atributos se desenvolvam e se tornem fonte de vantagem competitiva, é desejável a formação de um relacionamento de cooperação, seja qual for a sua intensidade, visando à complementaridade de recursos tangíveis e intangíveis para uma competitividade sustentada em longo prazo, possibilitando, assim, sua viabilidade econômica (Balestrin, 2005; Verschoore e Balestrin, 2006).

A indústria do turismo, mais especificamente, a do ecoturismo, foi considerada como base para a realização da pesquisa empírica proposta nesse trabalho. A escolha baseou-se na relevância e na potencialidade desse segmento para a economia brasileira, com forte influência no campo social, por meio da geração de renda e empregos formais. Somente em 2005, as atividades relacionadas ao turismo geraram quase $\mathrm{R} \$ 132$ bilhões de renda e mais de 8 milhões de postos de trabalho (Ibge, 2009).

A pesquisa de campo foi aplicada na região da Serra do Cipó, distante $100 \mathrm{~km}$ da capital mineira e localizada entre as históricas cidades de Ouro Preto e Diamantina. Considerada uma das maiores áreas de biodiversidade do planeta e um dos mais belos cenários de Minas Gerais (Descubra Minas, 2009), a região conta com o Parque Nacional Serra do Cipó e a terceira mais alta cachoeira do Brasil, atraindo 125 mil turistas por ano, interessados no ecoturismo (Ewi, 2007). Contando com quase uma centena de pousadas aglomeradas territorialmente, constituídas por micro e pequenas empresas, formou-se um ambiente propício à finalidade desse estudo.

Revista Ibero-Americana de Estratégia - RIAE, São Paulo, v. 10, n. 2, p. 34-57, mai./ago. 2011. 
Estratégia Cooperativa em Redes Organizacionais: Um Estudo em Polo Turístico de Minas Gerais

\section{REDES INTERORGANIZACIONAIS}

Segundo Peci (1999), o ambiente dos negócios está em constante transformação, sendo caracterizado por um cenário de incertezas e turbulências. A empresa hierárquica integrada verticalmente que caracterizou, por muito tempo, o modelo de produção da era fordista não atende mais às necessidades do novo paradigma organizacional. Diante disso, novas formas organizacionais, mais flexíveis, começam a proliferar, sendo as redes o elemento fundamental nesse novo processo. Para Silva Junior (2007), a aplicação dos conceitos de redes no contexto do atual ambiente de negócios decorre do aumento da competição entre empresas e da percepção de que as organizações, atuando de forma isolada, terão menos condições de sobrevivência e desenvolvimento. Isso porque nenhuma empresa domina sozinha os conhecimentos, recursos e habilidades necessárias para a sua sustentação. Da mesma forma, Verschoore (2004) destaca a importância das redes como instrumento para enfrentar as dificuldades e as crises do agitado ambiente competitivo e para permitir viabilizar ações que, isoladamente, não seriam tão facilmente alcançadas, tendo em vista a falta de poder competitivo de uma única empresa.

Casarotto Filho e Pires (2001) observam que a globalização da economia e os avanços tecnológicos, especialmente nas comunicações, são as principais causas das rápidas mudanças nos conceitos mercadológicos e de produção. Para os autores, a concorrência pode fabricar produtos melhores e mais baratos, estando em qualquer parte do mundo. Uma forma de diminuir tais ameaças e ganhar sinergia seria a formação de alianças entre empresas. Para Castells (1999), a integração em redes deve ser considerada como forma fundamental de concorrência na nova economia global. Nesse contexto, a cooperação e os sistemas de redes seriam a única forma de minimizar os riscos, de dividir os custos e de manter-se em dia com a informação constantemente atualizada. Ainda segundo Castells (1999), novas oportunidades são criadas o tempo todo quando se está dentro das redes. Mas, fora delas, a sobrevivência ficará cada vez mais difícil. Para Amato Neto (2000), as novas oportunidades de negócios tenderão a privilegiar produtos e serviços que envolvam alto conteúdo de conhecimento e de informações. A consequência disso é o surgimento de redes de cooperação produtivas entre as organizações. Casarotto Filho e Pires (2001) ratificam tal posição ao afirmar que o conhecimento será a grande mercadoria do futuro e que isso só terá valor onde existirem fluxos por meio de conexões, como é o caso das redes organizacionais.

De acordo com Vale (2007), não existe uma teoria única que trata de redes. São várias as discussões em torno desse assunto, com perspectivas bastante distintas, porém, com alguns pontos em comum, que permitem uma sistematização do tema. Para a autora, a evolução da abordagem de redes, no campo das ciências sociais, apresenta características peculiares, como a contribuição de

Revista Ibero-Americana de Estratégia - RIAE, São Paulo, v. 10, n. 2, p. 34-57, mai./ago. 2011. 
vários ramos de pensamento e o progresso do assunto em razão de uma forte interação entre teoria, método e pesquisa empírica. Silva Junior (2007) concorda com a abrangência e a complexidade do tema, mas afirma que a bibliografia sobre os relacionamentos entre empresas é fragmentada e influenciada por várias outras áreas de estudo, o que demonstra uma natureza multifacetada que envolve uma mescla de motivos, intenções e objetivos. Em uma tentativa de sistematizar a teoria sobre o assunto, sem, contudo, objetivar formar um modelo único ou identificar todas as teorias relacionadas, Silva Junior (2007) parte dos estudos de Barringer e Harrison (2000) e cita as principais abordagens sobre os relacionamentos interorganizacionais que compreendem as seguintes teorias: custo de transação, dependência de recursos, escolha estratégica, teoria do stakeholder, aprendizagem organizacional e teoria institucional.

Similar às abordagens teóricas, o conceito de redes também possui vários enfoques e está longe de chegar a uma unanimidade acadêmica. Para Cândido e Abreu (2000), até mesmo no sentindo etimológico, o termo "redes" pode conter vários significados. No entanto, os autores apontam para uma tendência do emprego do tema como caminho de se estudar as organizações e partem da ideia de que todas as organizações são formadas por redes sociais e, por isso, devem ser analisadas como tais. Nesse contexto, Amato Neto (2000), em uma primeira aproximação, conceitua o tema como um conjunto, ou uma série, de células interconectadas por relações bem definidas e prossegue, citando os estudos de Ribaut e Lebidois (1995), conforme citado em Amato Neto, (2000), que as redes de empresas consistem em um tipo de agrupamento cujo objetivo principal é o de fortalecer as atividades de cada um dos participantes da rede, sem que, necessariamente, tenham laços financeiros entre si. Para Castells (1999), uma rede é um conjunto de nós interconectados em que um nó depende do tipo de redes concretas no qual ele está inserido. Seguindo a mesma tendência, Lazzarini (2008) define redes como um conjunto de indivíduos ou organizações interligadas por meio de relações dos mais diversos tipos. Em um enfoque mais cooperativo, Jarillo (1988) explica o termo redes como um relacionamento de longo prazo entre organizações, com o propósito de sustentar uma posição competitiva em relação aos concorrentes que não fazem parte da rede. Carstens e Machado-da-Silva (2006) também descrevem as redes como estruturas formadas a partir da definição de papéis, atribuições e relações entre seus atores, o que permite a flexibilização do funcionamento da rede por intermédio das relações de cooperação.

Revista Ibero-Americana de Estratégia - RIAE, São Paulo, v. 10, n. 2, p. 34-57, mai./ago. 2011. 
Estratégia Cooperativa em Redes Organizacionais: Um Estudo em Polo Turístico de Minas Gerais

\subsection{ATUAÇÃO EM REDES DAS MPES}

As características inerentes às redes interorganizacionais podem servir como direcionamento das ações estratégicas de vários negócios, incipientes ou consolidados, pertencentes ao contexto das micro e pequenas empresas. Para Balestrin e Vargas (2004), são várias as dificuldades encontradas pelas MPEs no atual ambiente competitivo. Os autores citam como exemplo de tais dificuldades, o baixo volume de compras, que as impedem de obter melhores preços e vantagens; o alto custo de participação em feiras e campanhas publicitárias; os custos de reciclagem e o treinamento da mão de obra; a atualização tecnológica e o acesso à linhas de créditos e financiamentos. Conforme argumenta Balestrin e Vargas (2004), tais problemas dificilmente desaparecerão, mas podem ter seus efeitos neutralizados ou amenizados por meio das redes horizontais de cooperação. Para Tondolo e Schneider (2005), a formação de redes de pequenas empresas possibilita uma maior capacidade competitiva ao promover uma maior oferta de serviços e de produtos qualificados com o menor custo para o cliente, por meio do compartilhamento de informações, de tecnologia, de recursos, de oportunidades e, principalmente, de riscos.

Outro ponto importante a ser considerado é a confiança desenvolvida entre os atores como base para o desenvolvimento de relacionamentos colaborativos nas redes empresariais. De acordo com Amato Neto (2000), a confiança é o elemento central nas relações de cooperação e, segundo Balestrin e Vargas (2004), é fator crítico de sucesso paras as MPEs que, dificilmente, será alcançado por outras formas de redes entre grandes empresas e, muito menos, pelas grandes empresas integradas.

Casarotto Filho e Pires (2001) ressaltam ainda, que, a cooperação entre as pequenas empresas é algo tão irreversível quanto a globalização, e essa forma de atuação, talvez, seja a única maneira de assegurar a sobrevivência das MPEs.

\subsection{AGLOMERAÇÃO TERRITORIAL NO TURISMO}

Para Lastres e Cassiolato (2005), o termo "aglomeração" refere-se à proximidade territorial entre atores econômicos, políticos e sociais, podendo incluir empresas e outras organizações públicas e privadas, permitindo a formação de uma economia de aglomeração. Segundo os autores, tal economia é caracterizada pelas vantagens oriundas da proximidade geográfica dos atores, incluindo o acesso à conhecimentos, as capacitações, a mão de obra especializada, as matériasprimas e os equipamentos. Nesse contexto, a aglomeração constitui-se de uma relevante fonte geradora de vantagem competitiva, uma vez que as chances de sobrevivência e desenvolvimento 
das empresas aumentam consideravelmente, principalmente, no caso das MPEs. Andrighi e Hoffmamm (2008) concordam com o caráter vantajoso da aglomeração territorial e destacam outros benefícios encontrados nesse tipo de ambiente, como a redução do custo de transação, a criação de valor ao cliente, a redução de custos em pesquisas, a promoção do desenvolvimento local e o aumento do fluxo de informação. Para Verschoore (2003), o propósito central das aglomerações é reunir várias empresas em uma única estrutura apoiada em uma governança descentralizada que permita aos participantes manter a flexibilidade e a agilidade presentes nos pequenos negócios.

A aglomeração territorial parece não ser, somente, uma característica presente no setor de turismo e, sim, uma característica inerente a esse tipo de atividade. Segundo Petrocchi (2002), as atividades turísticas desenvolvem-se no entorno físico de cenários naturais, históricos, urbanos, culturais ou de lazer e crescem ao redor de atrativos turísticos, sempre constituídos por aglomerações geográficas de empresas. Nessa mesma linha, Beni (1998) afirma que a maioria dos produtos e serviços destinados ao turismo concentra-se em âmbitos geográficos bem definidos, onde se desenvolvem as atividades produtivas.

Para Andrighi e Hoffmamm (2008), os aglomerados territoriais no turismo são formados por diversos tipos de atores que possuem certa dependência típica para o desenvolvimento das complexas atividades relacionadas ao setor e, por esse motivo, destacam a importância da relação de cooperação entre os envolvidos como forma de se criar valor e manter uma vantagem competitiva. Com essa mesma perspectiva, Petrocchi (2002) reforça a ideia de cooperação para o progresso dos polos turísticos e salienta a exigência de uma forte complementaridade entre os diversos produtos e serviços para a satisfação final do turista. Ainda segundo o autor, o mau desempenho de uma das partes (atores) pode comprometer o desempenho do todo.

\subsection{APRENDIZADO RELACIONAL}

Segundo Hoffmann, Bandeira-de-Mello e Molina-Morales (2006), o interesse pela inovação em aglomerações territoriais na literatura acadêmica não é recente. Citam como exemplo o trabalho de Marshall (1925), que, ainda nas primeiras décadas do século XX, destacava que o conhecimento existente em determinada aglomeração perpetuava-se por meio de sua transferência de geração em geração, garantindo a formação de mão de obra das próprias empresas e do aglomerado como um todo. Com base em estudos mais recentes, os autores prosseguem com a análise do assunto e concluem que a inovação e a transferência do conhecimento são termos próximos e complementares no contexto das aglomerações e, que as empresas, direta ou indiretamente, transferem conhecimento entre si, estimulando o processo de inovação. A partir do instante em que a inovação é estabelecida,

Revista Ibero-Americana de Estratégia - RIAE, São Paulo, v. 10, n. 2, p. 34-57, mai./ago. 2011. 
Estratégia Cooperativa em Redes Organizacionais: Um Estudo em Polo Turístico de Minas Gerais

mesmo por algumas poucas empresas, ela é quase totalmente assimilada pelo aglomerado, que, em algum momento, passará a utilizá-la. No entanto, para que haja a transferência do conhecimento, é necessário que as empresas, isoladamente, aprendam de alguma forma. Para Nonaka e Takeuchi (1997), uma organização, sozinha, não pode criar conhecimento. É necessário que ela mobilize o conhecimento tácito criado e acumulado pelos indivíduos, de forma a expandir sua abrangência em níveis organizacionais e interorganizacionais. Isso é possível por intermédio dos quatro modos de conversão do conhecimento (socialização, externalização, combinação e internalização), o que permitirá a interação entre o conhecimento tácito e o explícito como maneira de elevar os níveis ontológicos. Desse ponto de vista, a criação do conhecimento organizacional nasce em um nível individual e se amplia para além das fronteiras organizacionais.

Balestrin e Fayard (2003) observam que o conhecimento interorganizacional, criado no âmbito de uma rede de empresas, é uma das mais amplas dimensões do conhecimento e que esse processo permite torná-lo mais completo, profundo e significativo. Entretanto, para que isso ocorra, é necessário um ambiente de sinergia para que a troca de experiências seja estimulada e continuada. Segundo os autores, esse modelo certamente não poderá ser reproduzido pelo ambiente tradicional das organizações, caracterizado pela rigidez e pela visão piramidal e, sim, por configurações organizacionais adaptadas a tal necessidade. Dessa demanda, destacam-se a redes interorganizacionais, capazes de proporcionar a efetiva interação entre pessoas, grupos e empresas, permitindo que o conhecimento, as práticas, os valores, o processo, a cultura e as diferenças individuais sejam compartilhados coletivamente. Molina-Morales e Hoffmann (2002) ratificam tal opinião ao dizer que a proximidade geográfica entre empresas é capaz de produzir uma interação social e profissional espontânea entre empreendedores e funcionários facilitando a difusão da informação e, consequentemente, do conhecimento.

\subsection{FRAGMENTAÇÃO DA REDE}

Mesmo em uma aglomeração territorial, com uma estrutura de atuação em redes já estabelecida, a fragmentação dos relacionamentos pode ocorrer. Vale (2007) reforça tal possibilidade ponderando que a proximidade física não é, isoladamente, fator determinante para a garantia de maior relacionamento e que, em um ambiente onde muitas empresas se especializam em produtos e serviços semelhantes e competem pelos mesmos clientes, a harmonia pode ser subestimada, dando lugar a um clima de competição e indiferença. Além disso, a atuação em redes exige esforços para serem estabelecidas e sustentadas (Andrade e Hoffmamm, 2010) demandando tempo e recursos que nem sempre são compartilhados em prol da coletividade, seja por falta de condições ou de interesse.

Revista Ibero-Americana de Estratégia - RIAE, São Paulo, v. 10, n. 2, p. 34-57, mai./ago. 2011. 
Maximiliano Francisco Oliveira \& Carlos Alberto Gonçalves

\subsection{PROPOSIÇÃO DO MODELO TEÓRICO DE VERIFICAÇÃO}

O objetivo proposto para essa pesquisa é descrever de que maneira a estrutura de relacionamento em redes das pousadas localizadas na região Serra do Cipó-MG determina a cooperação, o aprendizado relacional e a propensão a sua fragmentação ou desistência da cooperação. Resultante das considerações teóricas apontadas, esse trabalho se propõe verificar a veracidade de três hipóteses estruturadas pelos construtos mostrados no modelo hipotético da Figura 1. Assim, o modelo hipotético parte da premissa da atuação em redes entre as empresas aglomeradas territorialmente e considera que quanto maior for a intensidade desse tipo de relacionamento, maior será a cooperação entre os participantes (H1). Uma vez constatada a cooperação, pressupõe-se a existência do aprendizado relacional de forma proporcional à intensidade da cooperação (H2). Caso o processo da aprendizagem relacional enfraqueça ou não se forme, a tendência da rede é fragmentar-se (H3).

Figura 1- Modelo hipotético.

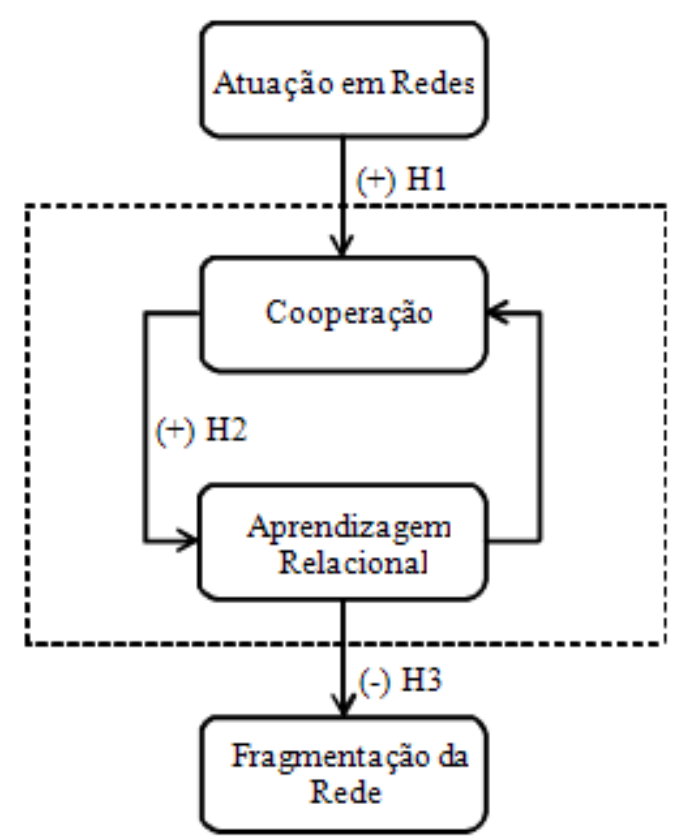

Revista Ibero-Americana de Estratégia - RIAE, São Paulo, v. 10, n. 2, p. 34-57, mai./ago. 2011. 
Estratégia Cooperativa em Redes Organizacionais: Um Estudo em Polo Turístico de Minas Gerais

\section{METODOLOGIA}

Como estratégia de pesquisa, foi utilizado o estudo de caso descritivo, que, de acordo com Gonçalves e Meirelles (2004), tem como finalidade investigar um fenômeno que deve apresentar certas características idiossincráticas iniciais e cujas unidades de análise e de observação, bem como o problema de pesquisa, devem limitar-se a um contexto específico. A natureza da pesquisa foi qualitativa com a utilização do método de survey. Tal método consiste em um questionário estruturado dado a uma amostra de população e destinado a provocar informações específicas dos entrevistados (Malhotra, 2001).

A área geográfica de abrangência dessa pesquisa foi a Serra do Cipó, com ênfase no município de Santana do Riacho, o mais importante receptivo da região. A população-alvo do estudo englobou as pousadas em funcionamento, constituídas por micro e pequenas empresas. A amostragem foi formada por adesão, com a participação de 19 pesquisados, selecionados de acordo com os seguintes critérios: 1) Estar localizado próximo à rodovia MG 10, entre os quilômetros 95 e 100, delimitados, respectivamente, pelo principal acesso ao Parque Nacional Serra do Cipó e pela cachoeira Véu da Noiva, uma das mais populares atrações da região. Nesse perímetro, concentra-se a maioria das pousadas e o comércio em geral, permitindo uma caracterização de aglomeração territorial; 2) Disponibilidade do proprietário da pousada, ou do seu preposto, em responder aos questionários de pesquisa. Considerando apenas o primeiro critério, estimou-se em 50 o número total de pousadas passíveis de serem entrevistadas. Portanto, a amostragem representou 38\% da população desejada.

Os dados primários foram coletados por meio de uma pesquisa de campo, realizada nos meses de dezembro de 2009 e janeiro de 2010, com a aplicação de um questionário fechado, contendo quatro perguntas, com opções de respostas baseadas na escala Likert e mais duas perguntas, com opções simples de respostas. O primeiro modo, procurou analisar os tipos de relacionamentos da pousada entrevistada com os demais participantes, sendo uma questão para cada construto do modelo hipotético. Para viabilizar tal modelo, a pesquisa foi dividida em duas fases consecutivas. Em um primeiro momento, foi realizado um contato, pessoalmente, com as pousadas, supostamente com o perfil desejado, em que foram apresentados a proposta da pesquisa e o convite de participação. Com a identificação das empresas dispostas em fazer parte do estudo, foi possível concluir a confecção das questões do questionário, contendo os nomes de todos os participantes, e agendar o início da segunda fase, que consistiu na sua aplicação. Quanto as demais questões, procurou-se identificar as características individuais de cada pesquisado e foram aplicadas, também, na segunda fase.

Revista Ibero-Americana de Estratégia - RIAE, São Paulo, v. 10, n. 2, p. 34-57, mai./ago. 2011. 
As respostas foram tabuladas em formato de matrizes relacionais (questões de 1 a 4) e tabelas com a totalização da frequência de cada resposta (questões 5 e 6). Por solicitação dos entrevistados, como forma de garantir o sigilo das respostas, as pousadas foram identificadas apenas por uma letra escolhida aleatoriamente entre A e S, sendo que as letras D, E, G, H, I, N, O, R e S representam as nove maiores pousadas, considerando o número total de funcionários.

As matrizes relacionais foram tratadas mediante utilização do software Ucinet 6.0, por meio de indicadores de tamanho, de fragmentação, de densidade e de coesão. O indicador tamanho representa o número total de ligações presentes entre os atores de determinado grupo de análise e permite verificar a existência de uma rede de relacionamento. No caso de um determinado ator não apresentar ligações com os demais, constata-se a fragmentação da rede (Borgatti, Everett e Freeman, 2002). O indicador densidade é calculado dividindo o número de ligações existentes pelo número máximo de ligações possíveis. Assim, quanto maior a ligação entre os atores, maior será a densidade da rede (Lazzarini, 2008). Com esse critério, foi possível mensurar a intensidade de cada construto proposto. O indicador coesão está relacionado à presença de subgrupos dentro de uma rede. Tais subgrupos caracterizam-se pela suposição da existência de um grau de afinidade entre os seus atores para que os relacionamentos possam ser estabelecidos. Uma rede é considerada coesa quando a quantidade de subgrupos for pequena ou inexistente.

Segundo Wasserman e Faust (1999), uma das possíveis formas de analisar a coesão de um subgrupo ocorre por meio da reciprocidade dos relacionamentos e da acessibilidade de um ator. A primeira abordagem indica a presença de grupos isolados e fortemente conectados, denominados de "cliques". A segunda abordagem, é uma ampliação do conceito da reciprocidade e considera também os atores não conectados diretamente entre si, mas que possuem ligações indiretas por meio de outros atores. Essa abordagem é também conhecida como n-clique, em que n representa o número máximo de intermediários necessários para acessar um determinador ator. Quanto menos cliques e n-cliques uma rede possuir, maior será sua coesão, e, consequentemente, maior será a potencialidade de desenvolver relacionamentos recíprocos.

Desse modo, com o intuito de verificar as hipóteses propostas, foi aplicada a análise de regressão estatística entre as matrizes relacionais. Segundo Malhotra (2001), tal técnica consiste em um processo de dedução de uma relação matemática entre uma única variável métrica dependente e uma única variável independente. Os cálculos foram realizados pelo software Microsoft Excel 2007 e serviram como parâmetro de mensuração dos relacionamentos.

Revista Ibero-Americana de Estratégia - RIAE, São Paulo, v. 10, n. 2, p. 34-57, mai./ago. 2011. 
Estratégia Cooperativa em Redes Organizacionais: Um Estudo em Polo Turístico de Minas Gerais

\section{APRESENTAÇÃO E ANÁLISE DOS DADOS}

\subsection{ANÁLISE DOS CONSTRUTOS}

As matrizes relacionais, originadas a partir das respostas dos questionários, possuem as mesmas características, com iguais números de linhas e colunas (matriz quadrada) e com idêntica composição de atores. Assim, pôde-se padronizar o método de análise com o emprego dos mesmos indicadores de redes para cada construto apresentado no modelo hipotético. Os cálculos dos indicadores basearam-se apenas na presença de laços não direcionais, que, segundo Lazzarini (2008), considera a possibilidade da reciprocidade dos relacionamentos em qualquer circunstância. Com esse critério, foi possível mensurar o tamanho potencial da rede pesquisada em 342 laços. Os indicadores, com seus respectivos resultados, são mostrados no Quadro 1.

\begin{tabular}{|c|c|c|c|c|}
\hline INDICADORES & $\begin{array}{c}\text { ATUAÇÃO EM } \\
\text { REDES }\end{array}$ & COOPERAÇÃO & $\begin{array}{l}\text { APRENDIZAGEM } \\
\text { RELACIONAL }\end{array}$ & $\begin{array}{c}\text { FRAGMENTAÇÃO } \\
\text { DA REDE }\end{array}$ \\
\hline Tamanho & 126 & 87 & 109 & 101 \\
\hline Fragmentação & 0 & 0 & 0 & 0 \\
\hline Densidade & $36,84 \%$ & $25,44 \%$ & $31,87 \%$ & $29,53 \%$ \\
\hline Subgrupo & Sim & Sim & Sim & Sim \\
\hline Clique & 23 & 6 & 12 & 63 \\
\hline 2-Cliques & 3 & 5 & 4 & 2 \\
\hline
\end{tabular}

Quadro 1- Quadro resumo dos indicadores de rede.

Ao analisar o tamanho das redes, foi possível verificar a inexistência de atores desconectados. Isso indica uma fragmentação igual a zero e demonstra que o total da amostra estudada se conecta direta ou indiretamente entre si. Com essa característica, confirmou-se a presença de todos os construtos entre as pousadas da Serra do Cipó e aceita-se a suposição inicial de atuação em redes. O quesito cooperação se destacou por possuir a rede de relacionamento mais coesa (Clique $=6$ ), porém, com a mais baixa densidade. A aprendizagem relacional alcançou a 
segunda maior densidade, superando a cooperação e a fragmentação da rede. Já a fragmentação da rede, se sobressaiu com a menor coesão no critério da reciprocidade dos relacionamentos (Clique = 63). Entretanto, com uma densidade maior que a cooperação e com um indicador 2-Cliques mais baixo entre os construtos (2-Cliques $=2$ ), supõem-se uma positiva tendência de crescimento.

A classificação da intensidade dos construtos baseou-se nos seguintes critérios: densidade da rede entre 0 e $30 \%$, baixa; entre 30 e $60 \%$, moderada; entre 60 e 100\%, alta. Portanto, observou-se uma moderada intensidade na atuação em redes e na aprendizagem relacional, enquanto na cooperação e na fragmentação da rede, a intensidade foi classificada como baixa.

Com o intuito de auxiliar e complementar a análise dos indicadores, foram geradas representações gráficas das redes de relacionamento. Dessa maneira, é possível visualizar o tamanho da rede, a existência da fragmentação e os indícios da densidade e como forma de complementação, identificar a composição dos subgrupos de atores. Os gráficos são compostos por nós e laços, sendo que o primeiro representa as empresas pesquisadas (atores), e o segundo, a existência de relacionamento.

Figura 2- Atuação em redes pelos atores sociais.

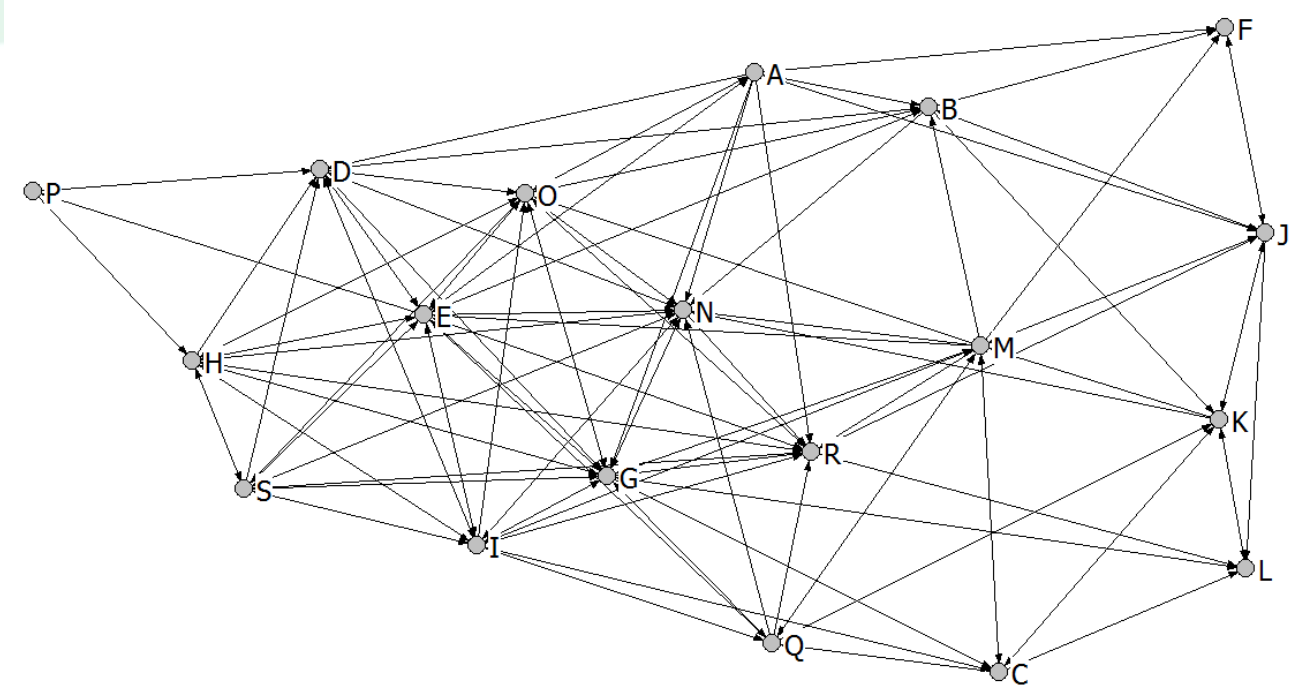

A Figura 2 mostra a estrutura de relacionamento referente ao construto atuação em redes e possibilita visualizar a conexão entre os dezenove participantes da pesquisa com a inexistência de fragmentação. Somando-se os laços, que representam os relacionamentos, encontra-se o tamanho da rede, com 126 ligações. A presença de atores periféricos, como por exemplo, o ator P, indica uma tendência de baixa densidade da rede. Quanto maior a quantidade de atores periféricos, maior a possibilidade de uma reduzida densidade. Ao analisar a coesão, é possível identificar a formação de um subgrupo predominante compostos pelos atores E, G, H, I, N, O, R e S.

Revista Ibero-Americana de Estratégia - RIAE, São Paulo, v. 10, n. 2, p. 34-57, mai./ago. 2011. 
Figura 3- Capacidade de cooperação pelos atores sociais.

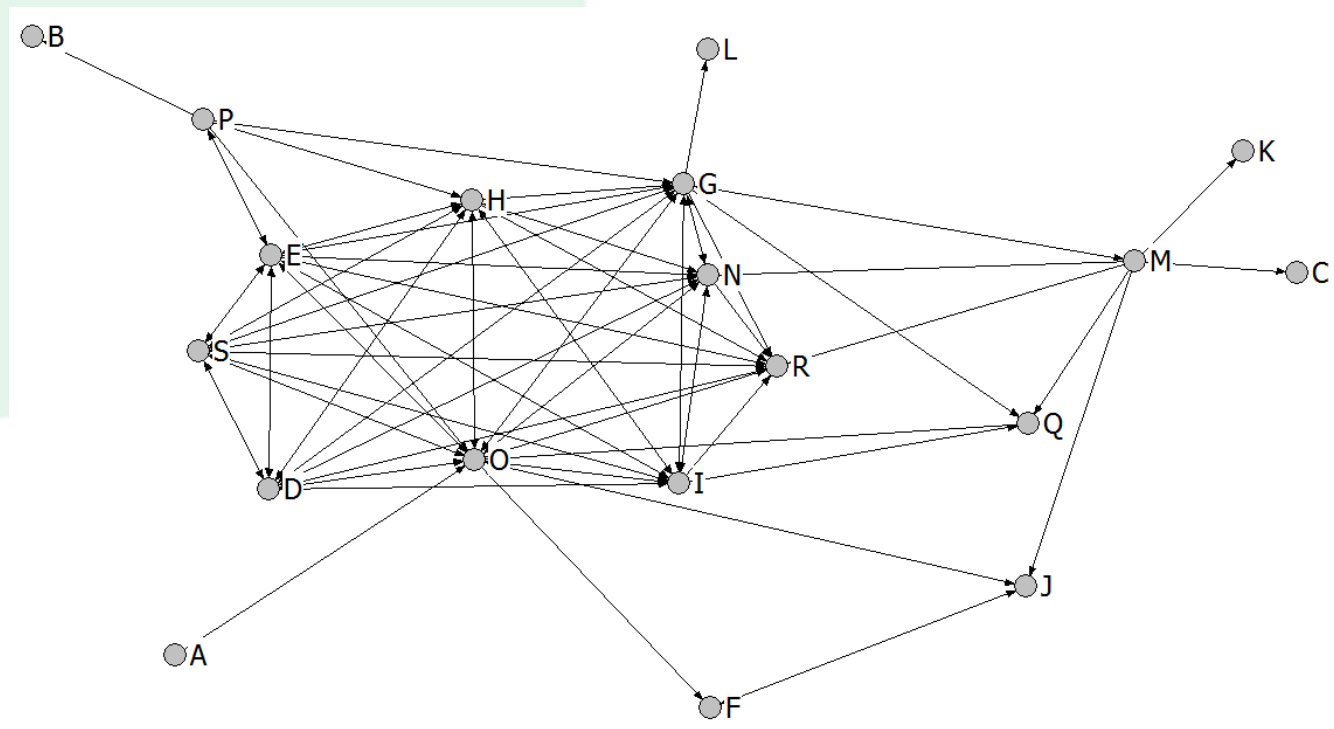

Ao visualizar a estrutura de relacionamento de cooperação, mostrada na Figura 3, percebe-se a conexão entre todos os atores. No entanto, com a presença de apenas 87 laços e com uma quantidade significativa de atores periféricos, observa-se a baixa densidade da rede. Quanto à coesão, verificou-se, também, a existência de um subgrupo principal, composto pelos atores $\mathrm{D}, \mathrm{E}$, G, H, I, N, O, R e S. Ao comparar a coesão da atuação em redes e da cooperação, é possível identificar os mesmos atores, entretanto, com uma predominância mais acentuada.

Figura 4 - Capacidade de aprendizagem relacional.

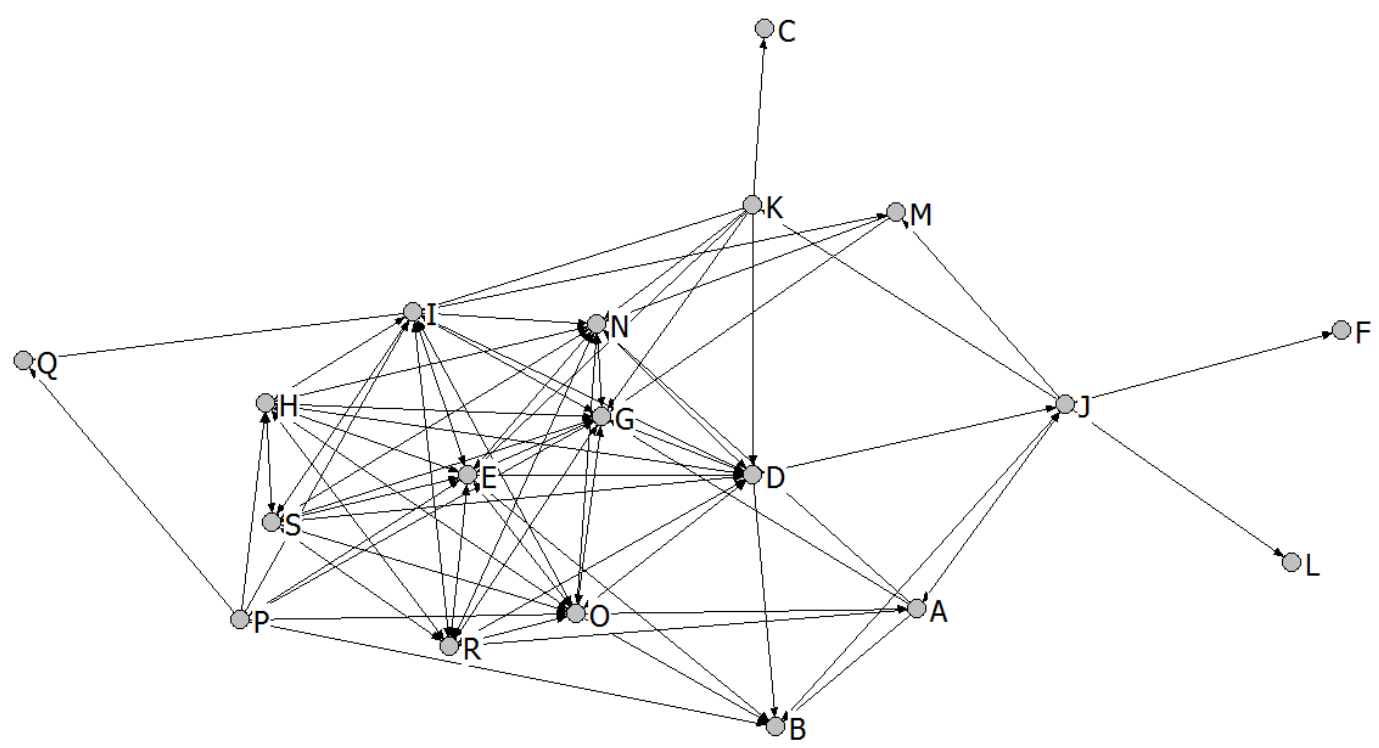

Revista Ibero-Americana de Estratégia - RIAE, São Paulo, v. 10, n. 2, p. 34-57, mai./ago. 2011. 
A estrutura da aprendizagem relacional é mostrada na Figura 4. Por intermédio dela, é possível observar o tamanho da rede, com 109 laços, conectando todos os atores. Em comparação com a rede de cooperação, observa-se que a rede de aprendizagem conta com um maior número de relacionamento e uma menor quantidade de atores periféricos, tornando-a mais densa. Seguindo a tendência das demais estruturas, constatou-se a presença do mesmo subgrupo predominante, formado pelos atores: D, E, G, H, I, N, O, R e S.

Figura 5 - Intenção de fragmentação da rede.

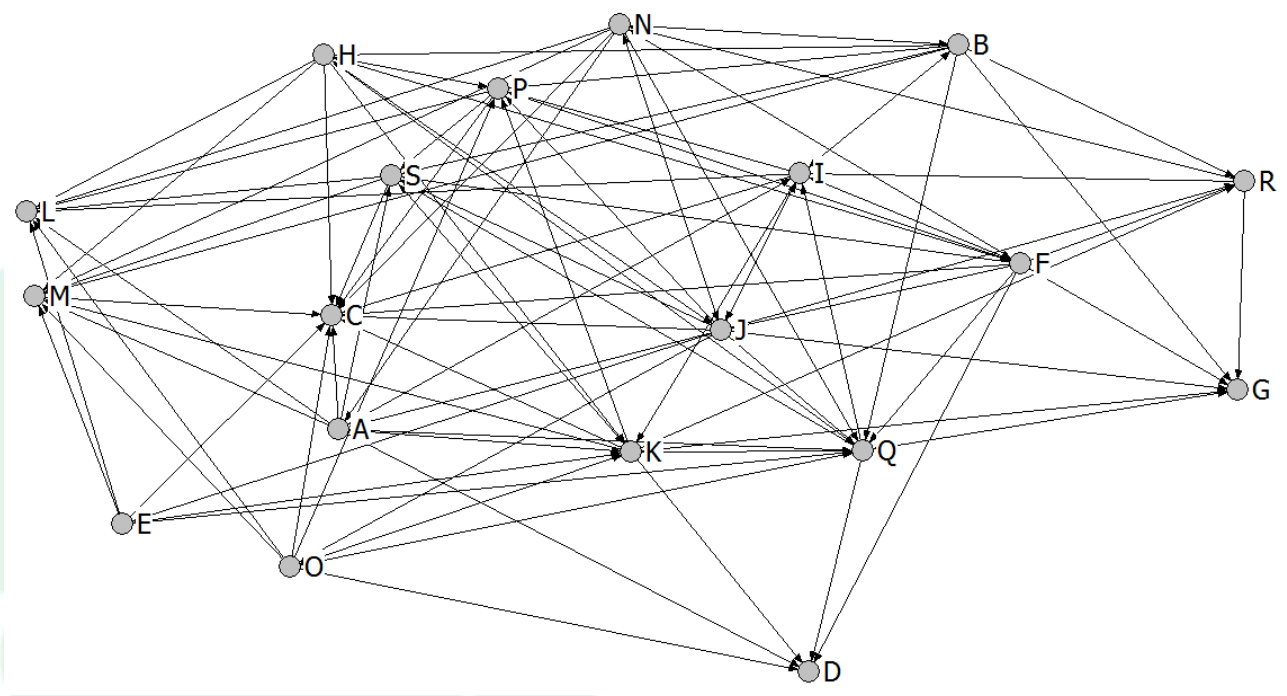

Ao observar a Figura 5, com a representação gráfica da estrutura de fragmentação da rede, percebe-se a ausência de atores isolados e a existência de 101 laços. Apesar dos atores periféricos não estarem tão evidentes quanto aos das figuras anteriores, é possível constatar a baixa densidade da rede pela formação de uma significativa quantidade de subgrupos (Clique $=63$ ).

\subsection{TESTE DO MODELO HIPOTÉTICO}

Objetivando testar o modelo hipotético, foi analisada a relação entre os construtos propostos como forma de encontrar indícios da aceitação, ou não, das hipóteses formuladas. Para isso, utilizou-se da análise de regressão estatística que se iniciou com a condensação das matrizes de relacionamento, somando-se os valores de cada coluna, de modo a formar uma linha totalizadora, que, posteriormente, foi dividida pelo número de pousadas participantes, chegando-se a uma média aritmética. A partir das médias encontradas, aplicou-se a análise de regressão linear, considerando as hipóteses de relação, cujos resultados são demonstrados na Quadro 2. 
Estratégia Cooperativa em Redes Organizacionais: Um Estudo em Polo Turístico de Minas Gerais

\begin{tabular}{|c|c|c|c|c|c|c|c|c|}
\hline HIPÓTESE & CONSTRUTO & $\mathbf{R 2}$ & $\begin{array}{c}\text { R2 } \\
\text { AJUSTADO }\end{array}$ & STAT T & GL & B1 & $\begin{array}{c}\text { VALOR } \\
\text { CRÍTICO DE } \boldsymbol{t}\end{array}$ & HIPÓTESE \\
\hline $\mathrm{H} 1$ & $\begin{array}{c}\text { Atuação em } \\
\text { Rede } \\
\text { Cooperação }\end{array}$ \\
$\mathrm{H} 2$ & $\begin{array}{c}\text { Cooperação } \\
\text { Aprendizagem } \\
\text { Relacional }\end{array}$ & 0,9159 & 0,8050 & 8,6793 & 17 & 0,8510 & 2,1098 & Não rejeitada \\
\hline $\mathrm{H} 3$ & $\begin{array}{c}\text { Aprendizado } \\
\text { Relacional } \\
\text { Fragmentação } \\
\text { da Rede }\end{array}$ & 0,2870 & 0,2450 & $(2,6156)$ & 17 & 0,7952 & 2,1098 & Não rejeitada \\
\hline
\end{tabular}

Quadro 2- Análise de regressão referente às relações do modelo hipotético.

Para cada hipótese, foi calculado o coeficiente de determinação mostrado na coluna r2 e r2 ajustado, que, de acordo com Malhotra (2001), mede a intensidade da associação entre duas variáveis, podendo resultar em um valor entre 0 a 1 . Quanto mais próximo de 1 for o coeficiente, maior será a relação. Os valores também podem variar negativamente, indicando uma relação inversa. O r2 ajustado difere do r2 por considerar o tamanho da amostra analisada. $\mathrm{O}$ teste de significância estatística da regressão linear deu-se por meio da comparação entre o valor calculado de $t$ e seu respectivo valor crítico. A estatística $t$ é utilizada para testar a hipótese nula de que não existe relação linear entre duas variáveis. Caso a hipótese seja nula (H0: $\beta=0)$ implica a inexistência de qualquer relação (Malhotra, 2001). O valor crítico de $t$ foi determinado pelo grau de liberdade $(\mathrm{gl})$ e por $\alpha=0,025(\mathrm{t}=2,1098)$ para um teste bicaudal. Dessa maneira, se $t$ for maior do que o seu valor crítico, rejeita-se a hipótese nula $(\mathrm{H} 0: \beta 1 \neq 0)$ e considera-se, como significante, a relação linear (hipótese não rejeitada). Caso contrário, quando $t$ for menor que o seu valor crítico, a hipótese é rejeitada.

Deste modo, constatou-se a relação positiva e significativa entre a atuação em redes e cooperação (H1), supondo que quanto maior a atuação em redes, maior a cooperação ( $\mathrm{r} 2=0,8159)$. A segunda hipótese $(\mathrm{H} 2)$ também foi aceita ao identificar que, quanto maior a cooperação, maior o aprendizado relacional $(\mathrm{r} 2=0,9100)$. A relação negativa entre o aprendizado relacional e a fragmentação da rede (H3) também foi identificada como significativa, porém, de forma menos intensa que os demais. Assim, pressupõe-se que, quanto menor o aprendizado, maior a tendência de fragmentação da rede.

Revista Ibero-Americana de Estratégia - RIAE, São Paulo, v. 10, n. 2, p. 34-57, mai./ago. 2011. 
Maximiliano Francisco Oliveira \& Carlos Alberto Gonçalves

\subsection{CARACTERIZAÇÃO DOS PARTICIPANTES DA PESQUISA}

Com o objetivo de analisar e descrever o perfil das pousadas participantes da pesquisa quanto ao número de colaboradores e ao tempo de atuação na região, o tamanho das empresas pertencentes à amostra e seu enquadramento na categoria das MPEs foi inicialmente identificado. Os critérios utilizados foram os mesmos adotados pelo Sebrae (2009) que classifica as empresas do setor de serviços, com até nove funcionários, de microempresas, e, entre 10 e 49 funcionários, de pequenas empresas. A Tabela 1 mostra os resultados da tabulação dos dados.

Tabela 1- Número de colaboradores.

\begin{tabular}{|c|c|c|c|}
\hline & MENOS DE 10 & ENTRE 10 E 49 & MAIS DE 49 \\
\hline Número de colaboradores & $53 \%$ & $47 \%$ & $0 \%$ \\
\hline
\end{tabular}

Quanto ao tempo de atuação, procurou-se observar o tempo em que cada pousada está atuando na região, de modo a encontrar indícios da possibilidade de atuação em redes, considerando o tempo de convivência entre elas. A tabulação dos dados coletados é mostrada na Tabela 2.

Tabela 2- Tempo de atuação.

\begin{tabular}{|c|c|c|c|}
\hline & MENOS DE 5 & ENTRE 6 E 10 & MAIS DE 10 \\
\hline Tempo de atuação (anos) & $26 \%$ & $37 \%$ & $37 \%$ \\
\hline
\end{tabular}

Ao analisar as tabelas, constatou-se que todas as pousadas possuem menos de 49 funcionários e que a maioria (74\%) conta com mais de cinco anos de atuação na Serra do Cipó. Com esse resultado, confirma-se, na amostragem selecionada, um aglomerado territorial de MPEs, com tempo suficiente de funcionamento para desenvolver atividades em rede. 
Estratégia Cooperativa em Redes Organizacionais: Um Estudo em Polo Turístico de Minas Gerais

\section{CONSIDERAÇÕES FINAIS}

Esse estudo objetivou analisar a estrutura de relacionamento em redes entre as pousadas localizadas na Serra do Cipó-MG, de forma a descrever de que maneira tal estrutura determina a cooperação e o aprendizado relacional. Para isso, foi selecionada uma amostragem de micro e pequenas empresas, aglomeradas territorialmente. Pretendeu-se, assim, verificar a existência, a intensidade, a potencialidade, bem como a relação dos construtos atuação em redes, cooperação, aprendizagem relacional e fragmentação da rede.

A partir da análise dos dados coletados, pôde-se confirmar a premissa inicial da atuação em redes entre as empresas estudadas e constatar que, quanto maior for essa característica, maior será a cooperação. Apesar da existência da cooperação, a sua baixa intensidade aponta para um baixo aproveitamento do seu potencial de crescimento, que é caracterizado pela expressiva coesão de sua estrutura e pela presença de um ambiente favorável, constituído por MPEs aglomeradas territorialmente, atuando em redes. Observa-se que a formação de um subgrupo predominante na estrutura de cooperação pode indicar um empecilho ao desenvolvimento das atividades cooperativas, uma vez que os esforços individuais tenderão a considerar grupos específicos, e não a rede como um todo. Um indício dessa premissa, baseia-se no fato dos subgrupos formados, na cooperação e na aprendizagem relacional, possuírem os mesmo atores.

Quanto ao aprendizado relacional, foi constatado uma significativa intensidade e o maior coeficiente de determinação (r2) em relação ao construto cooperação. Apesar disso, os dados analisados apontam para a presença do aprendizado relacional mesmo em situações de não cooperação. Tal fato se apoia pela intensidade do aprendizado ser maior que da cooperação. Desse modo, bastaria uma formação de aglomeração territorial no turismo para dar início ao processo de aprendizado. Fleury e Fleury (1997) ratificam essa possibilidade ao afirmarem que, com a observação das experiências realizadas por outras organizações, é possível constituir um importante caminho para o aprendizado. De maneira similar, Carstens e Machado-da-Silva (2006) utilizam-se do conceito de isomorfismo mimético como forma de viabilizar a transferência do conhecimento. Presume-se, portanto, independentemente da sua origem, o potencial positivo de crescimento da aprendizagem relacional.

Ao analisar a intenção de fragmentação da rede de relacionamento, observou-se que sua intensidade é superior à intensidade da cooperação. Tal fato indica para uma necessidade de maiores esforços no desenvolvimento e fortalecimento das atividades cooperativas. Considerando a suposição de quanto menor o aprendizado relacional, maior a tendência de fragmentação, o aprendizado poderia ser um eficiente meio de incremento para a convergência da rede.

Revista Ibero-Americana de Estratégia - RIAE, São Paulo, v. 10, n. 2, p. 34-57, mai./ago. 2011. 
Apesar dos esforços na realização da pesquisa de campo e nas análises dos dados coletados, as seguintes limitações foram observadas: 1) A unidade de análise foi restrita e específica da Serra do Cipó, não permitindo generalizar as conclusões obtidas para outras destinações turísticas ou demais setores com características de aglomeração territorial; 2) A amostra da pesquisa foi escolhida por adesão e representa apenas parte das pousadas atuantes na região, significando que as opiniões dos pesquisados não representam, necessariamente, a unanimidade da população. Dessa maneira, sugere-se a realização de novos estudos, na mesma região e em outros aglomerados turísticos, de modo a se procurar novos indícios de padrões de comportamentos interorganizacionais. Uma pesquisa comparativa entre dois ou mais aglomerados também seria bastante útil para novas conclusões.

\section{REFERÊNCIAS}

Amato Neto, J. (2000). Redes de cooperação produtiva e clusters regionais: oportunidades para as pequenas e médias empresas. São Paulo: Atlas.

Andrade, M. A. R. , Hoffmann, V. E. (2010). Redes interorganizacionais: um estudo das pequenas e médias empresas no setor calçadista do Vale do Rio Tijucas. Revista de Administração e Inovação, São Paulo, 7( 2), 193-216.

Andrighi, F. F. \& Hoffmamm, V. E. (2008, jul.). Aglomeração territorial no turismo: uma avaliação dos atores sociais na destinação turística de Urubici/SC. Cultur: Revista de Cultura e Turismo, Santa Cruz, 2.

Balestrin, A. (2005). A dinâmica da complementaridade de conhecimentos no contexto das redes interorganizacionais. 2005. 214 f. Tese (Doutorado) Programa de Pós-Graduação em Administração, Universidade Federal do Rio Grande do Sul, Porto Alegre.

Balestrin, A. \& Fayard, P. (2003). Redes interorganizacionais como espaço de criação de conhecimento. In XXVII Encontro da Associação Nacional de Pós-Graduação e Pesquisa em Administração. Anais, Atibaia: ANPAD.

Balestrin, A. \& Vargas, L. M. (2004). A Dimensão estratégica das redes horizontais de PMEs: teorizações e evidências. Revista de Administração Contemporânea. 8,203-227.

Barringer, B. R. \& Harrison, J. S. (2000). Walking a tightrope: creating value through interorganizational relationships. Journal of Management. 26(3), 367-403.

http://dx.doi.org/10.1177/014920630002600302

Revista Ibero-Americana de Estratégia - RIAE, São Paulo, v. 10, n. 2, p. 34-57, mai./ago. 2011. 
Beni, M. C. (1998). Análise estrutural do turismo. (2 ed.) São Paulo: Senac.

Borgatti, S. P., Everett, M. G \& Freeman, L. C. (2002). UCINET 6 for Windows: Version 6.199. Natick: Analytic Technologies.

Brasil. IBGE - Instituto Brasileiro de Geografia e Estatística (2008). Economia do turismo: Uma perspectiva macroeconômica 2000-2005. Recuperado em 30 de abril, 2009, de <http://www.ibge.gov.br>.

Cândido, G. A. \& Abreu, A. F. (2000). Os conceitos de redes e as relações interorganizacionais: um estudo exploratório. In EnANPAD, 24. Florianópolis. Anais, Florianópolis: ANPAD.

Casarotto Filho, N. \& Pires, L. H. (2001). Redes de pequenas e médias empresas e desenvolvimento local: estratégias para a conquista da competitividade global com base na experiência italiana. (2 ed.) São Paulo: Atlas.

Carstens, D. D. S., Machado-da-Silva, C. (2006). Estratégia e estrutura de relacionamento na rede de empresas Alpha. In XXX Encontro da Associação Nacional de Pós-Graduação e Pesquisa em Administração. Anais, Salvador: ANPAD.

Castells, M. (1999). A sociedade em rede. (2 ed.) São Paulo: Paz e Terra..

Descubra Minas (Minas Gerais). Senac - MG. (2009). Recuperado em 4 de maio, 2009, de <http://www.descubraminas.com.br/>.

Ewi - Eplerwood International (Brasil). Instituto Estrada Real. (2007). Diagnóstico turismo de natureza: Destino Serra do Cipó Estrada Real. Minas Gerais. Brasil. Disponível. Belo Horizonte,. 142 p. Recuperado em 1 de maio, 2009, de <http://www.estradareal.org.br>.

Ferreira Junior, I. , Teixeira, R. M. (2007). Redes de pequenas empresas: a aplicação de uma tipologia em uma rede de supermercados. Revista de Administração Mackenzie. 8(3), 128-152.

Fleury, A. C. C. , Fleury, M. T. L. (1997). Aprendizagem e inovação organizacional: as experiências de Japão, Coreia e Brasil.(2 ed.) São Paulo: Atlas.

Gonçalves, C. A, Gonçalves Filho, C. \& Reis Neto, M. T. (2006). Estratégia empresarial: o desafio das organizações. São Paulo: Saraiva.

Gonçalves, C. A \& Meirelles, A. M. (2004). Projetos e relatórios de pesquisa em administração. São Paulo: Atlas, 1.

Hitt, M. A, Ireland, R. D. \& Hoskisson, R. E. (2008). Administração estratégica.( 2 ed.) São Paulo: Cengage.

Revista Ibero-Americana de Estratégia - RIAE, São Paulo, v. 10, n. 2, p. 34-57, mai./ago. 2011. 
Hoffmann, V. E., Bandeira-de-Melo, R., Molina-Morales, F. X. (2006). Inovação e transferência de conhecimento em redes inter-organizacionais aglomeradas territorialmente: uma análise a partir de equações estruturais em duas indústrias. In XXX Encontro da Associação Nacional de PósGraduação e Pesquisa em Administração. Anais, Salvador: ANPAD.

Hoffmann, V. E, Molina-morales, F. X \& Martínez-Fernadez, M. (2004). T. Redes de empresas: Uma tipologia para sua classificação. In XXVIII Encontro da Associação Nacional de PósGraduação e Pesquisa em Administração. Anais, Curitiba: ANPAD.

Jarillo, J. C. (1988, jan-feb.). On strategic networks. Strategic Management Journal, 31-41. http://dx.doi.org/10.1002/smj.4250090104

Lastres, H. M. M. e Cassiolato, J. E. (2005). Mobilizando conhecimentos para desenvolver arranjos e sistemas produtivos e inovativos locais de micro e pequenas empresas no Brasil. Rede de pesquisa em sistemas produtivos e inovativos locais. Recuperado em 23 de setembro, 2009, de <http://www.redeaplmineral.org.br/biblioteca/>.

Lazzarini, S. G. (2008). Empresas em rede. São Paulo: Cengage Learning.

Malhotra, N. K. (2001). Pesquisa de marketing: uma orientação aplicada. (3. ed.) Porto Alegre: Bookman.

Marshall, A. (1925). Principles of economics. (8.ed.) London: Macmillan.

Matheus, R. F e Silva, A. B. O. E. (2006). Análise de redes sociais como método para a Ciência da Informação. Datagramazero: Revista da Ciência da Informação, Rio de janeiro, 7(2).

Molina-Morales, F. X. e Hoffmann, V. E. (2002, jul.). Aprendizagem através de redes sociais: o efeito da proximidade geográfica. Revista Inteligência Empresarial, Rio de Janeiro, 12, 4-11.

Nonaka, I. e Takeuchi, H. (1997). Criação de conhecimento na empresa: como as empresas japonesas geram a dinâmica da inovação. Rio de Janeiro: Campus.

Peci, A. (1999). Emergência e proliferação de redes organizacionais - Marcando mudanças no mundo de negócios. In XXIII Encontro da Associação Nacional de Pós-Graduação e Pesquisa em Administração. Anais, Foz do Iguaçu: ANPAD.

Petrocchi, M. (2002). Gestão de pólos turísticos. São Paulo: Futura.

Porter, M. E. (1998, nov-dec). Clusters and the new economics competition. Havard Business Review, p. 77-90.

Ribaut, M., Martinet, B. \& Lebidois, D. (1995). A gestão das tecnologias. Coleção gestão \& inovação. Lisboa: Publicações Dom Quixote. 
Sebrae. Serviço Brasileiro de Apoio às Micro e Pequenas Empresas. (2009). Recuperado em 22 de novembro, 2009, de <http://www.sebrae.com.br/customizado/estudos-e-pesquisas>.

Silva Junior, A. B. (2007). A empresa em rede: desenvolvendo competências organizacionais. Rio de Janeiro: Elsevier.

Tondolo, V. A. G e Schneider, L. C. (2005). Vantagens logísticas na cadeia de valor na rede de empresas: o caso de uma rede gaúcha de pequenas farmácias. Revista Alcance. 18( 3), 143-160.

Vale, G. M. V. (2007). Territórios vitoriosos: o papel das redes organizacionais. Rio de Janeiro: Garamond.

Verschoore, J. R. (2003, out). O programa redes de cooperação: uma análise dos instrumentos de administração pública para o desenvolvimento sócio-econômico. VIII Congresso Internacional del CLAD sobre la Reforma del Estado y de la Administración Pública, Panamá. Recuperado em 21 de setembro, 2009, de < http://www.unpan.org>.

Verschoore, J. R. (2004). Redes de cooperação: uma nova organização de pequenas e médias empresas no Rio Grande do Sul. Porto Alegre: FEE.

Verschoore, J. R e Balestrin, A. (2006). Fatores competitivos das empresas em redes de cooperação. In: XXX Encontro da Associação Nacional de Pós-Graduação e Pesquisa em Administração. Anais, Salvador: ANPAD.

Wasserman, S. e Faust, K. (1999). Social network analysis: methods and applications. Cambridge: Cambridge University Press.

Recebido: 03/05/2011

Aprovado: 23/07/2011

Revista Ibero-Americana de Estratégia - RIAE, São Paulo, v. 10, n. 2, p. 34-57, mai./ago. 2011. 